\title{
Calificación de riesgo operatorio en cirugía de tórax no cardíaca
}

\author{
Dagho Domínguez Olguín,凶 Octavio Narváez Porras
}

Servicio de Cirugía de Tórax, Instituto Nacional de Enfermedades Respiratorias Ismael Cosío Villegas, Ciudad de México, México.
Trabajo recibido: 04-X-2013; aceptado: 30-I-2014

RESUMEN. La valoración del riesgo quirúrgico es indispensable al someter a los pacientes a un procedimiento para evitar complicaciones perioperatorias. Los tiempos largos de espera para realizar dicha valoración originan un retraso importante en la resolución quirúrgica. Se realizó una actualización de la valoración convencional (Goldman) agregando los índices de riesgo pulmonar, renal y cardíaco con el fin de mejorar la predicción de morbimortalidad, agrupándolos en una única valoración integral (CARIOPE) con un resultado final por sumatoria de puntajes de donde se obtuvieron 3 niveles de riesgo con significancia estadística. Se realizó un estudio prospectivo, observacional y descriptivo aplicando la CARIOPE a cada paciente sometido a cirugía no ambulatoria no cardíaca del 01 septiembre de 2010 al 18 mayo de 2012 , con seguimiento los primeros 30 días posquirúrgicos y documentando la morbimortalidad presentada en cada nivel de riesgo. El análisis estadístico se realizó con PASW statics v.18 con un total de 1,097 pacientes, morbilidad de $12.3 \%$ y mortalidad de $3.3 \%$. Se obtuvieron valores estadísticamente significativos en la predicción de complicaciones cardíacas, pulmonares y renales, entre otras $(p<0.05)$. La CARIOPE es un instrumento eficaz de determinación de riesgo perioperatorio en diferentes aparatos y sistemas y no solamente en el área cardíaca.

Palabras clave: Valoración preoperatoria, índices de riesgo quirúrgico, complicación posquirúrgica, morbimortalidad.

\begin{abstract}
Assessment of surgical risk is essential when submitting patients to a procedure in order to avoid perioperative complications. Lengthy wait times in performing such assessment create important delays for surgical solution. An updating of the conventional assessment (Goldman) was performed by incorporating lung, kidney and cardiac risk rates to improve prediction of morbidity and mortality, merging them into a single, comprehensive assessment (CARIOPE- Spanish initials for Perioperative Risk Rating), obtaining a final score by adding individual rates, which yielded three statistically significant risk levels. A prospective, observational, and descriptive study was conducted, applying CARIOPE to each patient who underwent non- ambulatory, non-cardiac surgery from September 1, 2010 to May 18, 2012, with a patient follow up during the first 30 postoperative days to document morbidity and mortality in each risk level. Statistical analysis was performed using PASW statistics v.18 yielding a total of 1,097 patients, a morbidity of $12.3 \%$, and a mortality of $3.3 \%$. Statistically significant values were obtained for the prediction of cardiac, lung, and kidney complications, among others $(p<0.05)$. CARIOPE is an effective tool for the assessment of perioperative risk in various organ systems and is not confined to cardiac risk.
\end{abstract}

Key word: Preoperative assessment, surgical risk index, surgical complications, morbidity and mortality rate.

\section{INTRODUCCIÓN}

Los tiempos actuales requieren de una mejora en la calidad de la atención médica y en la disminución de costos para mejorar la economía, tanto del paciente como de las instituciones de salud. Todo paciente intervenido quirúrgicamente requiere de una valoración preoperatoria para identificar el riesgo de desarrollo de complicaciones perioperatorias y que pueden ser prevenibles. En nuestro medio, existe la práctica de solicitar una valoración preoperatoria por parte del especialista en medicina interna, esto impacta en un tiempo promedio de espera de dos meses en los pacientes manejados por consulta externa y cuatro días en pacientes hospitalizados, lo que eleva el costo hospitalario. Más de $55 \%$ de estas valoraciones son realizadas en pacientes que no presentan ninguna comorbilidad, y son solicitadas únicamente porque el paciente es mayor de 40 años como requisito en nuestro medio bajo una interpretación de las Normas Oficiales Mexicanas 170 y 205., ${ }^{1,2}$

Las complicaciones que se presentan con mayor frecuencia son en los sistemas: respiratorio, ${ }^{3-5}$ cardiovascular ${ }^{6-8}$ y renal, ${ }^{9,10}$ entre otros; y la valoración convencional de Goldman ${ }^{11}$ sólo identifica riesgos en el área cardíaca, la cual tiene más de 20 años desde su publicación, al presente existen actualizaciones y mejoras de la misma. ${ }^{12,13}$

Nos dimos a la tarea de buscar en la literatura índices de riesgo en las áreas que presentan la mayor morbi- 
mortalidad, encontrando índices de riesgo pulmonar (PRI, del inglés pulmonary risk index [tabla 1]), ${ }^{11}$ índice de riesgo cardíaco revisado en cirugía de tórax (ThR$\mathrm{CRI}$, del inglés thoracic revised cardiac risk index [tabla 2]), ${ }^{13}$ los predictores de falla renal aguda (PARF, del inglés predictors of acute renal failure [tabla 3] $)^{10} \mathrm{y}$ la valoración de la Sociedad Americana de Anestesiología (ASA, del inglés American Society of Anesthesiologists [tabla 4]), ${ }^{14}$ las cuales son sencillas y fáciles de aplicar, además, pueden ser realizados por el personal médico quirúrgico sin necesidad de una interconsulta inicial por el especialista en medicina interna. Se realizó la sumatoria de la calificación de cada índice de riesgo descrito

Tabla 1. Índice de riesgo pulmonar.

\begin{tabular}{|c|c|c|}
\hline $\begin{array}{l}\text { Obesidad } \\
\text { Tabaquismo últimas } 8 \text { sema- }\end{array}$ & $\begin{array}{l}1 \text { punto } \\
1 \text { punto }\end{array}$ & \multirow{6}{*}{ PRI } \\
\hline Tos productiva últimos 5 días & 1 punto & \\
\hline $\begin{array}{l}\text { Estertores difusos últimos } 5 \\
\text { días }\end{array}$ & 1 punto & \\
\hline $\mathrm{FEV}_{1} / \mathrm{FVC}<70 \%$ & 1 punto & \\
\hline $\mathrm{PaCO}_{2}>50$ & 1 punto & \\
\hline EPOC & 1 punto & \\
\hline Grados & $\begin{array}{l}\text { Puntaje } \\
\text { obtenido }\end{array}$ & $\begin{array}{c}\% \text { de } \\
\text { complicación } \\
\text { pulmonar }\end{array}$ \\
\hline 1 & 0 a 1 & 4.1 \\
\hline 2 & 2 a 3 & 9 \\
\hline 3 & 4 a 5 & 30 \\
\hline 4 & $>5$ & 46 \\
\hline
\end{tabular}

Modificado de Chest 1993;104(3):694-700.

Abreviaturas: PRI: Índice de riesgo pulmonar; FEV : Volumen espiratorio forzado en el primer segundo; FVC: Capacidad vital forzada; $\mathrm{PaCO}_{2}$ : Presión parcial de bióxido de carbono en sangre arterial; EPOC: Enfermedad pulmonar obstructiva crónica. en una valoración única a la que llamamos Calificación de Riesgo Operatorio o CARIOPE (tabla 5).

La finalidad del estudio fue aplicar la CARIOPE a todos los pacientes sometidos a cirugía para identificar el riesgo perioperatorio, y según el resultado realizar la intervención operatoria si los riesgos son bajos o solicitar la interconsulta convencional a los médicos especialistas si los riesgos son intermedios o altos, según el sistema que resulte comprometido en la valoración (renal, cardíaco, respiratorio, etc.). ${ }^{15,16}$ Con ello se pretende disminuir el porcentaje de complicaciones al detectar pacientes con riesgos aumentados, y disminuir la estancia hospitalaria prolongada (la cual se define como una estancia igual o mayor a 14 días) ${ }^{17}$ en espera de una valoración preoperatoria convencional; y por ende, disminuir los costos tanto a la institución como al paciente y familiares. Servirá también como medio informativo tanto para el paciente como para los médicos en la firma del consentimiento informado valorando el riesgo-beneficio de las intervenciones médicas y quirúrgicas. ${ }^{18-21}$

\section{MATERIAL Y MÉTODOS}

Se utilizaron los PARF, PRI, ThRCRI y la clasificación de la ASA realizando una sumatoria del resultado de cada índice, agrupándolos en un solo índice de riesgo llamado CARIOPE (tabla 5) dando un puntaje mínimo de 4 y máximo de 18, dividiéndolos en 3 grupos de riesgo (bajo, intermedio y alto). Se incluyeron a todos los pacientes sometidos a cirugía en el Servicio de Cirugía Torácica del Instituto Nacional de Enfermedades Respiratorias Ismael Cosío Villegas de la Ciudad de México, en el período comprendido de septiembre de 2010 a mayo de 2012, excluyendo pacientes en cirugía ambulatoria y aquellos a los que no fue posible dar el

Tabla 2. Clasificación de ThRCRI para riesgo cardíaco.

\begin{tabular}{|c|c|c|c|}
\hline $\begin{array}{l}\text { Enfermedad cerebrovascular } \\
\text { Isquemia cardíaca } \\
\text { Enfermedad renal o creatinina }>2 \text { o filtración } \\
\text { glomerular }<60 \\
\text { Neumonectomía programada }\end{array}$ & $\begin{array}{l}1.5 \text { puntos } \\
1.5 \text { puntos } \\
1 \text { punto } \\
1 \text { punto }\end{array}$ & 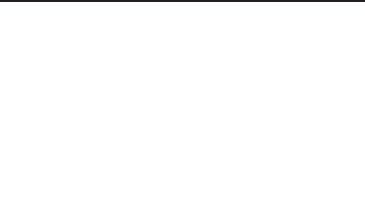 & ThRCRI \\
\hline WN/W. & Puntaje obtenido & $\begin{array}{c}\text { Complicación cardíaca } \\
\text { mayor }(\%)\end{array}$ & $\begin{array}{l}\text { Mortalidad de origen } \\
\text { cardíaco (\%) }\end{array}$ \\
\hline $\begin{array}{l}1 \\
2 \\
3 \\
4\end{array}$ & $\begin{array}{c}0 \\
1 \text { a } 1.5 \\
2 \text { a } 2.5 \\
>2.5\end{array}$ & $\begin{array}{l}1.5 \\
5.8 \\
19 \\
23\end{array}$ & $\begin{array}{c}0.9 \\
4.2 \\
8 \\
18\end{array}$ \\
\hline
\end{tabular}

Modificado de: Ann Thorac Surg 2011;92(2):445-448.

Abreviatura: ThRCRI: Índice de riesgo cardíaco revisado en cirugía torácica. 
Tabla 3. Clasificación de PARF para riesgo renal.

\begin{tabular}{|c|c|c|}
\hline $\begin{array}{l}\text { Edad mayor a } 59 \text { años } \\
\text { Cirugía de urgencia } \\
\text { Enfermedad hepática } \\
\text { Índice de masa corporal > } 32 \mathrm{~kg} / \mathrm{m} 2 \\
\text { Cirugía de alto riesgo (tórax, abdo- } \\
\text { men, vascular inguinal) Estimado alto } \\
\text { de pérdidas hemáticas }\end{array}$ & $\begin{array}{l}1 \text { punto } \\
1 \text { punto } \\
1 \text { punto } \\
1 \text { punto } \\
1 \text { punto }\end{array}$ & PARF \\
\hline Grados & $\begin{array}{l}\text { Puntaje } \\
\text { obtenido }\end{array}$ & $\begin{array}{c}\% \text { de } \\
\text { falla renal } \\
\text { aguda }\end{array}$ \\
\hline 1 & 0 & 0.30 \\
\hline 2 & 1 & 0.50 \\
\hline 3 & 2 & 1.30 \\
\hline 4 & 3 o más & 4.30 \\
\hline
\end{tabular}

Modificado de: Anesthesiology 2007;107(6):892-902.

Abreviatura: PARF: Predictores de falla renal aguda.

Tabla 4. Clasificación de la ASA para determinar estado físico preoperatorio.

Clasificación de la ASA

\begin{tabular}{|c|c|}
\hline Grados & $\begin{array}{l}\text { Clasificación del estado funcional } \\
\text { preoperatorio }\end{array}$ \\
\hline 1 & Paciente sano \\
\hline 2 & Enfermedad sistémica moderada \\
\hline 3 & $\begin{array}{l}\text { Enfermedad sistémica severa que limita la } \\
\text { actividad, pero no incapacita }\end{array}$ \\
\hline 4 & $\begin{array}{l}\text { Enfermedad sistémica incapacitante que } \\
\text { compromete la vida }\end{array}$ \\
\hline 5 & $\begin{array}{l}\text { Paciente moribundo que se espera no } \\
\text { sobreviva las siguientes } 24 \text { horas }\end{array}$ \\
\hline 6 & Programa de donación de órganos \\
\hline$E$ & Se agrega en caso de urgencia \\
\hline
\end{tabular}

Modificado de: AANA J 2003;71(4):265-274.

Abreviatura: ASA: Sociedad Americana de Anestesiología. seguimiento durante los primeros 30 días posquirúrgicos. Los pacientes que resultaron con riesgo intermedio y alto fueron enviados a valoración preoperatoria y tratamiento preoperatorio por especialistas en medicina interna, cardiología y nefrología, principalmente. A las cirugías de urgencia también se les realizó la CARIOPE, y si las condiciones lo permitían también se interconsultó de manera urgente a la especialidad de medicina interna y terapia intensiva. El análisis de datos se realizó con el programa PASW statics v. 18 midiendo pruebas estadísticas para variables nominales y ordinales a través de Pearson Chi-Square y Fisher's Exact Test, tomando como significancia estadística una p menor a 0.05 y realizando tablas cruzadas y análisis univariados y multivariados. Para determinar los puntajes para cada nivel de riesgo de la CARIOPE se realizaron las mismas pruebas estadísticas que determinaron una $p$ menor a 0.05 entre cada nivel de riesgo (figura 1), existiendo esta diferencia entre los puntajes 7 y 8 determinando el nivel de riesgo bajo e intermedio y entre los puntajes 12 y 13 determinando el nivel de riesgo intermedio y alto (figuras 1-3). Se realizaron análisis estadísticos entre cada nivel de riesgo de CARIOPE comparándolas estadísticamente contra las complicaciones y mortalidad obtenidas. El estudio fue aprobado por el comité de ética en investigación de la institución.

\section{RESULTADOS}

Obtenidos de un total de 1,097 pacientes (tabla 6). Los diagnósticos más frecuentes fueron: empiema, $15.57 \%$; estenosis traqueal, $10.66 \%$; y tumores pulmonares, $10.47 \%$. Las cirugías más frecuentes fueron: lavado y decorticación, $18.3 \%$; biopsias pulmonares, $15.3 \%$; y fibrobroncoscopias, $11.1 \%$. El porcentaje de cirugías fue: menores, $50.18 \%$; mayores, $45.45 \%$; y urgencia, $4.37 \%$.

Tabla 5. Calificación CARIOPE para determinar las probabilidades de morbimortalidad perioperatoria.

\begin{tabular}{lccc}
\hline Índice de riesgo & Grado obtenido & & \\
ASA & 4 a 6 puntos & \\
ThRCRI & 1 a 4 puntos & CARIOPE \\
PRI & 1 a 4 puntos & & \\
PARF & 1 a 4 puntos & Probabilidad de desarrollar & Probabilidad de \\
& Sumatoria del puntaje & complicaciones (\%) & 0.5 \\
Riesgo & obtenido & 7.6 & 6 \\
\hline Bajo & De 4 a 7 puntos & 19.4 & 57.1 \\
Intermedio & De 8 a 12 puntos & 66.7 & (1) \\
Alto & 13 o más puntos & & \\
\hline
\end{tabular}

CARIOPE: Calificación de riesgo operatorio. 

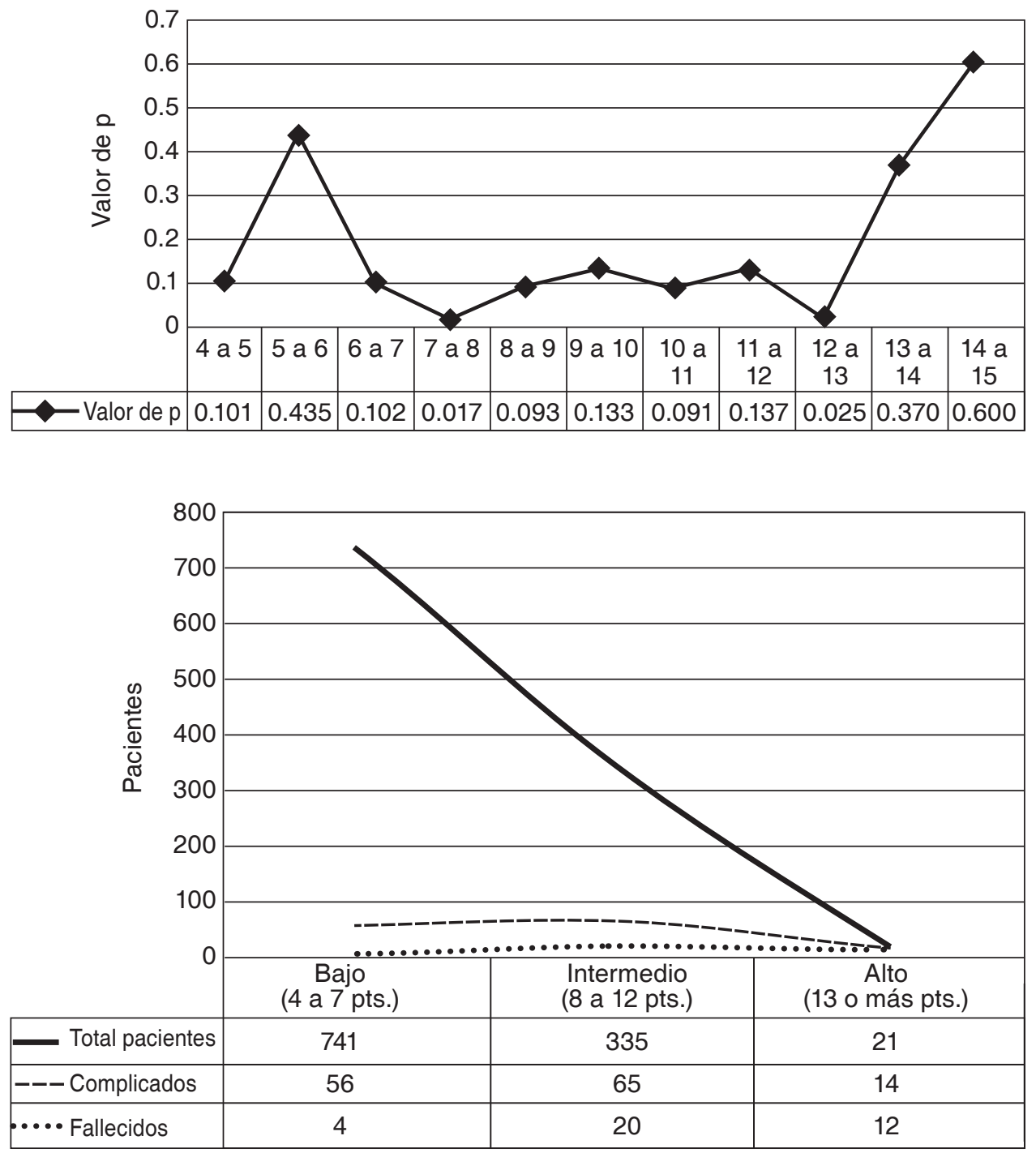

Se toma valor de $\mathrm{p}<0.05$ como significativamente estadístico.

\section{Figura 1.}

Valor de $\mathrm{p}$ comparando cada una de las puntuaciones obtenidas de la calificación de riesgo operatorio.
Figura 2.

Total de pacientes en cada nivel de riesgo de la calificación de riesgo operatorio.

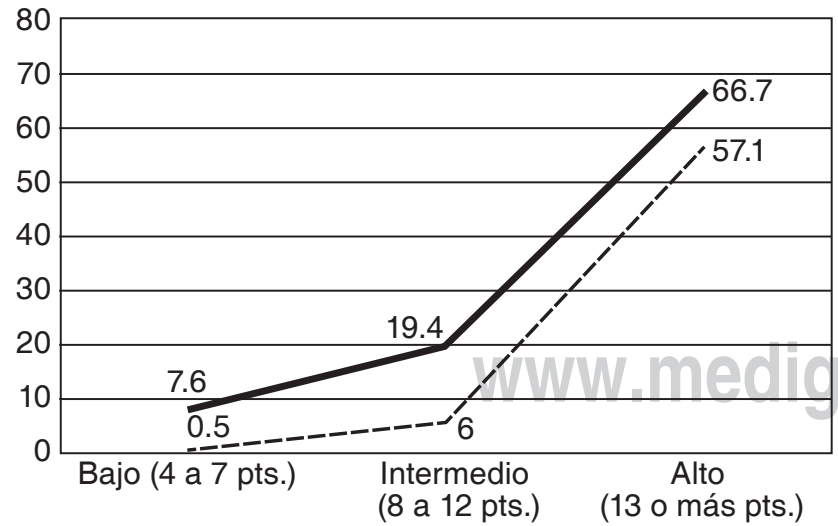

Porcentaje de complicados

--- Porcentaje de fallecidos

Figura 3. Porcentaje de morbimortalidad obtenido por cada nivel de riesgo de la calificación de riesgo operatorio.
Tabla 6. Resultados estadísticos generales.

Edad promedio
Mínima
Máxima
Masculino
Femenino

42.85 años

1 mes

101 años

$63 \%$

$37 \%$

El porcentaje de complicaciones fue del $12.3 \%$. Las 3 principales complicaciones fueron en general: hemotórax posquirúrgico (11.76\%), infección de herida quirúrgica $(9.80 \%)$ y neumotórax $(9.15 \%)$. La mortalidad fue de $3.3 \%$ y las principales causas de mortalidad fueron, en general, insuficiencia respiratoria, sepsis y falla orgánica múltiple.

Los resultados por cada nivel de riesgo de la CARIOPE arrojaron una buena significancia estadística 
Tabla 7. Resultados de tablas cruzadas valorando el porcentaje de predicción de cada grado de la CARIOPE con respecto a los sistemas que resultaron con significancia estadística en el análisis univariado $(p<0.05)$.

\begin{tabular}{|c|c|c|c|c|c|c|c|}
\hline \multirow[b]{2}{*}{$\begin{array}{l}\text { Nivel de riesgo } \\
\text { CARIOPE }\end{array}$} & \multicolumn{7}{|c|}{ Complicaciones por sistema } \\
\hline & Cardíaco & Pulmonar & Renal & Sepsis & FOM & $\begin{array}{l}\text { Insuficiencia } \\
\text { respiratoria }\end{array}$ & $\begin{array}{c}\text { Estancia hospitalaria } \\
\text { prolongada }\end{array}$ \\
\hline Bajo & $0.3 \%$ & $1.3 \%$ & $0.3 \%$ & $0 \%$ & $0.1 \%$ & $0.5 \%$ & $7.3 \%$ \\
\hline Intermedio & $2.7 \%$ & $6 \%$ & $1.5 \%$ & $1.8 \%$ & $0.9 \%$ & $1.8 \%$ & $10.5 \%$ \\
\hline Alto & $19 \%$ & $9.5 \%$ & $14.3 \%$ & $28.6 \%$ & $4.8 \%$ & $4.8 \%$ & $26.3 \%$ \\
\hline Valor de $p$ & 0.000 & 0.000 & 0.000 & 0.000 & 0.003 & 0.035 & 0.000 \\
\hline
\end{tabular}

CARIOPE: Calificación de riesgo operatorio; FOM: Falla orgánica múltiple.

Estancia hospitalaria prolongada 14 o más días.

en la predicción de complicaciones y de la mortalidad (tabla 5), dando resultados de morbilidad del $7.6 \%$ y mortalidad de $0.5 \%$ en el nivel de riesgo bajo que corresponde de 4 a 7 puntos obtenidos por la sumatoria de los índices de riesgo pulmonar, cardíaco, renal y ASA; en el riesgo intermedio que corresponde de 8 a 12 puntos, la morbilidad fue de $19.4 \%$ y mortalidad de $6 \%$; y en el riesgo alto que corresponde de 13 o más puntos, la morbilidad fue de $66.7 \%$ y mortalidad de $57.1 \%$. El análisis estadístico entre cada nivel de riesgo arrojó una $p<0.001$. Asimismo, se obtuvo una buena significancia estadística en la predicción de complicaciones en los siguientes sistemas por separado: pulmonares, cardíacas, renales, falla orgánica múltiple, insuficiencia respiratoria y sepsis (tabla 7 ).

\section{DISCUSIÓN}

La CARIOPE es de utilidad para la identificación de factores de riesgo en pacientes que serán sometidos a un procedimiento quirúrgico con una adecuada significancia estadística en la predicción de la morbilidad para complicaciones cardíacas, pulmonares, renales, falla orgánica múltiple, sepsis, estancia hospitalaria prolongada, insuficiencia respiratoria y mortalidad. Es sencilla de aplicar y sirve de apoyo para identificar pacientes con altos riesgos que ameritan una valoración extra por especialistas en medicina interna, cardiología o nefrología, según el área afectada previo a su intervención quirúrgica.

Al presente, estamos en la segunda fase analizando los resultados de morbimortalidad de pacientes con valoración convencional contra pacientes con CARIOPE; lo ideal es encontrar una mejoría en la identificación de riesgos y disminución en el porcentaje de pacientes con estancia hospitalaria prolongada en pacientes con CARIOPE. Es necesario realizar valoraciones externas para reproducirlas en otras áreas diferentes a las que realizamos en cirugía de tórax no cardíaca como la cirugía general o en medicina general. Es una herramienta útil para la firma del consentimiento informado, determinando el riesgo que tiene cada paciente de desarrollar complicaciones en el perioperatorio sumado a las complicaciones esperadas por el propio procedimiento quirúrgico. También sirve de apoyo al valorar el riesgo-beneficio de la terapia quirúrgica contra el tratamiento médico en pacientes con altos riesgos. El único estudio que probablemente no se tenga en todos los hospitales es la espirometría que usamos en un punto en la identificación de riesgo pulmonar $\mathrm{PRI}(\mathrm{FEV} / \mathrm{FVC})$, el cual podría suplirse con prueba de ejercicio dando positivo con desarrollo de disnea a leves esfuerzos o una historia clínica de disnea a los medianos a leves esfuerzos. Los demás estudios son básicos en cualquier laboratorio y gabinete hospitalario.

\section{REFERENCIAS}

1. Norma Oficial Mexicana. Para la práctica de anestesiología. NOM-170-SSA1-1998.

2. Norma Oficial Mexicana. Para la práctica de la cirugía mayor ambulatoria. NOM-205-SSA1-2002.

3. Seely AJ, Ivanovic J, Threader J, et al. Systematic classification of morbidity and mortality after thoracic surgery. Ann Thorac Surg 2010;90(3):936-942.

4. Kim JB, Lee SW, Park SI, Kim YH, Kim DK. Risk factor analysis for postoperative acute respiratory distress syndrome and early mortality after pneumonectomy: the predictive value of preoperative lung perfusion distribution. J Thorac Cardiovasc Surg 2010;140(1):26-31.

5. Ferguson MK, Gaissert HA, Grab JD, Sheng S. Pulmonary complications after lung resection in the absence of chronic obstructive pulmonary disease: the predictive role of diffusing capacity. J Thorac Cardiovasc Surg 2009;138(6):1297-1302.

6. Wiklund RA, Stein HD, Rosenbaum SH. Activities of daily living and cardiovascular complications following elective, noncardiac surgery. Yale J Biol Med 2001;74(2):75-87.

7. van Klei WA, Bryson GL, Yang H, Kalkman CJ, Wells GA, Beattie WS. The value of routine preoperative electro- 
cardiography in predicting myocardial infarction after noncardiac surgery. Ann Surg 2007;246(2):165-170.

8. Lee TH, Marcantonio ER, Mangione CM, et al. Derivation and prospective validation of a simple index for prediction of cardiac risk of major noncardiac surgery. Circulation 1999;100(10);1043-1049.

9. Licker M, Cartier V, Robert J, et al. Risk factors of acute kidney injury according to RIFLE criteria after lung cancer surgery. Ann Thorac Surg 2011;91(3):844-851.

10. Kheterpal S, Tremper KK, Englesbe MJ, et al. Predictors of postoperative acute renal failure after noncardiac surgery in patients with previously normal renal function. Anesthesiology 2007;107(6):892-902.

11. Epstein SK, Faling LJ, Daly BD, Celli BR. Predicting complications after pulmonary resection. Preoperative exercise testing vs. a multifactorial cardiopulmonary risk index. Chest 1993;104(3);694-700.

12. Brunelli A, Varela G, Salati M, et al. Recalibration of the revised cardiac risk index in lung resection candidates. Ann Thorac Surg 2010;90(1):199-203.

13. Brunelli A, Cassivi SD, Fibla J, et al. External validation of the recalibrated thoracic revised cardiac risk index for predicting the risk of major cardiac complication after lung resection. Ann Thorac Surg 2011;92(2):445-448.

14. Aronson WL, McAuliffe MS, Miller K. Variability in the American society of anesthesiologists physical status classification scale. AANA J 2003;71(4):265-274.

15. Jaffer AK, Smetana GW, Cohn S, Slawski B. Perioperative medicine update. J Gen Intern Med 2009;24(7):863-871.

16. Hernandez AF, Newby LK, O'Connor CM. Preoperative evaluation for major noncardiac surgery: focusing on heart failure. Arch Intern Med 2004;164(16):1729-1736.
17. Seely AJ, Ivanovic J, Threader J, et al. Systematic classification of morbidity and mortality after thoracic surgery. Ann Thorac Surg 2010;90(3):936-942.

18. Ivanovic J, Al-Hussaini A, Al-Shehab D, et al. Evaluating the reliability and reproducibility of the Ottawa Thoracic Morbidity and Mortality classification system. Ann Thorac Surg 2011;91(2):387-393.

19. Ferguson MK, Gaissert HA, Grab JD, Sheng S. Pulmonary complications after lung resection in the absence of chronic obstructive pulmonary disease: the predictive role of diffusing capacity. J Thorac Cardiovasc Surg 2009;138(6):1297-1302.

20. Jeger RV, Probst C, Arsenic R, et al. Long-term prognostic value of the preoperattive 12-lead electrocardiogram before major noncardiac surgery in coronary artery disease. Am Heart J 2006;151(2):508-513.

21. Shapiro M, Swanson SJ, Wright CD, et al. Predictors of major morbidity and mortality after pneumonectomy utilizing the Society for Thoracic Surgeons General Thoracic Surgery Database. Ann Thorac Surg 2010;90(3):927-934.

\section{$\triangle$ Correspondencia:}

Dr. Dagho Domínguez Olguín

Servicio de Cirugía, Instituto Nacional de

Enfermedades Respiratorias Ismael Cosío Villegas.

Calzada de Tlalpan 4502, colonia Sección XVI.

México, D.F., 14080

Correo electrónico: dagho12@gmail.com

Los autores declaran no tener conflictos de interés. 